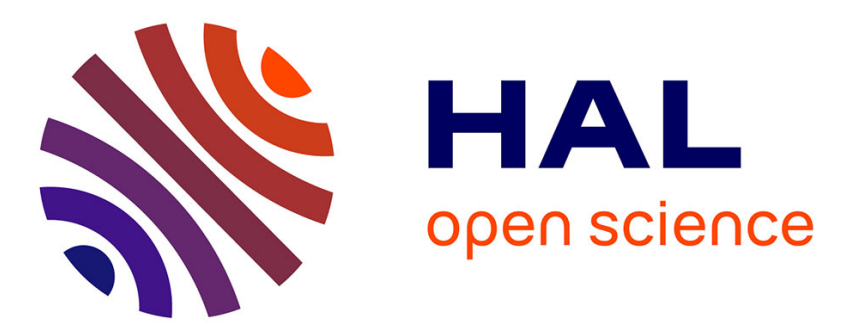

\title{
Scattering from quasi-planar and moderate rough surfaces: Efficient method to fill the EFIE-Galerkin MoM impedance matrix and to solve the linear system
} Christophe Bourlier

\section{- To cite this version:}

Christophe Bourlier. Scattering from quasi-planar and moderate rough surfaces: Efficient method to fill the EFIE-Galerkin MoM impedance matrix and to solve the linear system. IEEE Transactions on Antennas and Propagation, 2021, 69 (9), pp.5761 - 5770. 10.1109/TAP.2021.3060920 . hal-03120958

\section{HAL Id: hal-03120958 \\ https://hal.science/hal-03120958}

Submitted on 30 Jun 2021

HAL is a multi-disciplinary open access archive for the deposit and dissemination of scientific research documents, whether they are published or not. The documents may come from teaching and research institutions in France or abroad, or from public or private research centers.
L'archive ouverte pluridisciplinaire HAL, est destinée au dépôt et à la diffusion de documents scientifiques de niveau recherche, publiés ou non, émanant des établissements d'enseignement et de recherche français ou étrangers, des laboratoires publics ou privés. 


\title{
Scattering from quasi-planar and moderate rough surfaces: Efficient method to fill the EFIE-Galerkin MoM impedance matrix and to solve the linear system
}

\author{
Christophe Bourlier
}

\begin{abstract}
First, an acceleration to compute the impedance matrix obtained from the EFIE (electric field integral equation) discretized by the Galerkin MoM (method of moments) with RaoWilton-Glisson basis functions, is addressed. It is based on a farfield approximation and makes it possible to avoid to loop on the source and observation triangles. Next, the impedance matrix is split into strong and weak interactions; the latter is compressed by expressing it from Toeplitz sub-matrices. Then, the linear system is efficiently solved by a bi-iterative scheme. For a given order, the LSQR (least squares QR) algorithm is applied to solve the sparse linear system related to the strong interactions, while the matrix-vector products, related to the weak interactions, are accelerated by using FFTs. Numerical results of the field scattered by perfectly-conducting paraboloid-shape object and Gaussian rough surface are shown.

Index Terms-Electric field integral equation, Method of moments, Radar cross section, Fast algorithm, Rough surface scattering.
\end{abstract}

\section{INTRODUCTION}

The calculation of full vector wave scattering from a large perfectly-conducting surface is a very challenging issue; the main difficulty lies in the problem size. Solving a problem of this kind, from the EFIE (electric field integral equation) discretized by the Galerkin MoM (method of moments) with RaoWilton-Glisson basis functions [1], requires a great number of unknowns $N_{\text {Edge }}$ in order to obtain accurate and meaningful results. Thus, reduction of both computation time and data storage requirement is continuously in progress.

Direct solvers, like the LU decomposition, require $\mathcal{O}\left(N_{\text {Edge }}^{3}\right)$ operations while iterative solvers [2], such as conjugate gradient or generalized minimal residual (GMRES) techniques [3], need $\mathcal{O}\left(N_{\text {Edge }}^{2}\right)$ operations for the matrix-vector multiplication at each iteration. The memory requirement for these two solvers is usually $\mathcal{O}\left(N_{\text {Edge }}^{2}\right)$. Such computational complexity and memory requirements are too restrictive to be able to solve a large-scale scattering problem.

These two disadvantages can be alleviated by using the SMCG (sparse matrix canonical grid method) [4], [5], [6], [7], the adaptive integral method (AIM) [8], [9], [10], [11], [12], the SEBCM (stabilized extended boundary condition method) [13] and the FMM (fast multipole method) [14],

C. Bourlier is with the IETR (Institut d'Electronique et des Technologies numéRique), UMR CNRS 6164, University of Nantes, France (e-mail: christophe.bourlier@univ-nantes.fr).
[15]. For far-field interactions, the principle of AIM and SMCG is similar and consists in expressing the Green function on a uniform grid by using an interpolation scheme. From SMCG, a Taylor series expansion is also applied on the surface elevations. It is related to the concept of the short interaction range [16]. This implies that the matrix is Toeptliz, making it possible to reduce the memory requirement and to accelerate the matrix-vector product by using FFTs. The first novelty of this paper is to accelerate the calculation of the weak impedance matrix elements by deriving a closed-form expression, resulting from the sum over the 4 facets common to the source and observation edges. In addition, since with a triangular discretization the basis functions are not uniformly distributed, the weak impedance matrix is expressed from 3 Toeplitz sub-matrices by defining two uniform sub-grids. This avoids applying interpolations. Next, for quasi-planar surfaces and similarly to [7], the surface elevation $\Delta z$ is accounted for by expanding the Toeplitz sub-matrices over $\Delta z$.

For an iterative solver, the convergence of iterations is not very good for ill-posed matrix equations. This often occurs when the EFIE is used to solve 3-D complex scattering problems. For instance, Hu et al. [17] added the MFIE contribution to decrease the condition number of the resulting matrix, and the linear system is solved from a bi-iterative procedure. The present method distinguishes the strong near-field interactions and the weak ones between the observation point and the source point. This has the advantage that only the near interaction matrix is stored and the far interaction matrix is Toeplitz by blocks and requires to store only $\mathcal{O}\left(N_{\text {Edge }}\right)$ elements. The second novelty of this paper is to take advantage of this decomposition for solving efficiently the linear system from a bi-iterative algorithm. Then, for a given order, the LSQR (least squares QR) algorithm [18] is applied to efficiently solve the sparse linear system related to the strong interactions, while the matrix-vector products, related to the weak interactions, are accelerated by using FFTs. Numerical tests showed that LSQR converges more rapidly than GMRES or bi-conjugate gradient stabilized method.

The paper is organized as follows. Section II presents the EFIE impedance matrix and the scattered field. Section III addresses the derivation of the weak interaction impedance matrix and section IV deals with how this matrix can be partitioned into Toeplitz sub-matrices. Section V presents the bi-iterative scheme to efficiently solve the linear system and 
section VI shows numerical results for a paraboloid-shaped object and a Gaussian rough surface. The last section gives concluding remarks.

\section{EFIE IMPEDANCE MATRIX AND SCATTERED FIELD}

In this paper, to compute the field scattered by a perfectlyconducting object, the EFIE is solved from the MoM. In addition, the Galerkin method is applied by using the RaoWilton-Glisson basis functions. This leads to solve the linear system $\bar{Z} \boldsymbol{X}=\boldsymbol{b}$, where $\bar{Z}$ is the impedance matrix and $\boldsymbol{X}$ a vector related to the incident wave. The time convention $e^{-j \omega t}$ is used throughout this paper.

The element $Z_{m, n}$ of the impedance matrix $\bar{Z}$, corresponding to the interaction between two edges $m$ (observation) and $n$ (source) of a facet couple $(p, q)$, is expressed as [2]

$$
\begin{aligned}
Z_{m, n}=\frac{s_{p} s_{q}}{4 \pi A_{p} A_{q} L_{m} L_{n}} \iint_{\mathrm{T}_{p}} \iint_{\mathrm{T}_{q}} & \left(\boldsymbol{\rho}_{m}^{p} \cdot \boldsymbol{\rho}_{n}^{q}-\frac{1}{k^{2}}\right) \\
& \times \frac{e^{-j k D_{p, q}}}{D_{p, q}} d R_{p} d R_{q},
\end{aligned}
$$

where $s_{p, q}= \pm 1,\left\{A_{p, q}\right\}$ the triangle areas, $\left\{L_{m, n}\right\}$ the edge lengths, $\boldsymbol{\rho}_{m, n}^{p, q}=\left(\boldsymbol{V}_{m, n}^{p, q}-\boldsymbol{R}_{p, q}\right) / 2$, in which $\boldsymbol{V}_{m, n}^{p, q}$ is the position vector of the vertex unshared by the edge $(m, n)$ and belonging to the facet $(p, q)$. In addition, $D_{p, q}=\left\|\boldsymbol{R}_{p}-\boldsymbol{R}_{q}\right\|$ and $k$ is the wavenumber which equals $2 \pi / \lambda$, where $\lambda$ is the wavelength in free space.

Assuming a plane incident wave, a component $b_{n}$ of the vector $\boldsymbol{b}$ associated to the source edge $m$ and facet $p$ is given by [2]

$$
b_{m}=-\frac{j}{\omega \mu} \frac{L_{m} s_{m}}{2 A_{p}} \iint_{\mathrm{T}_{p}} \boldsymbol{\rho}_{m}^{p} \cdot \hat{\boldsymbol{p}}_{\text {inc }} e^{-\boldsymbol{k}_{\mathrm{inc}} \cdot \boldsymbol{R}_{p}} d R_{p},
$$

where $\omega$ is the wave pulsation and $\mu$ the permeability of the surrounding medium. In addition, $\hat{\boldsymbol{p}}_{\text {inc }}$ (either vertical, $\hat{\boldsymbol{v}}_{\text {inc }}$ $(\theta)$, or horizontal, $\left.\hat{\boldsymbol{h}}_{\text {inc }}(\phi)\right)$ and $\boldsymbol{k}_{\text {inc }}$ are the polarization and incident wave vectors, respectively, both defined in spherical coordinates from the angles $\left(\theta_{\text {inc }}, \phi_{\text {inc }}\right)$. By solving the linear system $\boldsymbol{X}=\overline{\boldsymbol{Z}}^{-1} \boldsymbol{b}$, the components $\left\{a_{n}\right\}$ of the vector $\boldsymbol{X}$ are found. The scattered far field is then expressed as

$$
\begin{aligned}
\boldsymbol{E}_{\text {sca }}^{\infty}\left(\boldsymbol{R}_{0}\right) & =-\frac{j \omega \mu e^{-j k R_{0}}}{8 \pi R_{0}} \sum_{p=1}^{P_{\text {Facet }}} \sum_{m=1}^{M_{\text {Edge }}} \frac{L_{m} a_{m} s_{m}}{A_{p}} \\
& \times \iint_{\mathrm{T}_{p}} \boldsymbol{\rho}_{m}^{p} e^{j \boldsymbol{k}_{\text {sca }} \cdot \boldsymbol{R}_{p}} d R_{p},
\end{aligned}
$$

where $P_{\text {Facet }}$ is the number of facets and $M_{\text {Edge }}$ the number of edges associated to the facet $p$. In addition, $R_{0}$ is the distance from the receiver to the phase origin of the object. The scattering coefficient is then expressed as

$$
\mathrm{SC}_{p_{\text {inc }} p_{\text {sca }}}=\lim _{R_{0} \rightarrow \infty} 2 \sqrt{\pi} R_{0} \frac{\boldsymbol{E}_{\text {sca }}^{\infty} \cdot \hat{\boldsymbol{p}}_{\text {sca }}}{\boldsymbol{E}_{\text {inc }} \cdot \hat{\boldsymbol{p}}_{\text {inc }}},
$$

where $p_{\text {inc }}=\{\theta, \phi\}$ and $p_{\text {sca }}=\{\theta, \phi\}$. The subscripts "inc" and "sca" stand for incident and scattered (waves), respectively. The receiver polarization basis $\left(\hat{\boldsymbol{k}}_{\text {sca }}, \hat{\boldsymbol{v}}_{\text {sca }}, \hat{\boldsymbol{h}}_{\text {sca }}\right)$ can be defined in a similar way as that of the incident field $\left(\hat{\boldsymbol{k}}_{\text {inc }}, \hat{\boldsymbol{v}}_{\text {inc }}, \hat{\boldsymbol{h}}_{\text {inc }}\right)$, in which $\theta_{\text {sca }}$ and $\phi_{\text {sca }}$ are the receiver (scattering) angles. The radar cross section $\mathrm{RCS}_{p_{\text {inc }} p_{\text {sca }}}$ is obtained by taking the squared modulus of $\mathrm{SC}_{p_{\text {inc }} p_{\text {sca }}}$.

\section{DERIVATION OF THE WEAK INTERACTION IMPEDANCE MATRIX}

The impedance matrix is split into near $\bar{Z}_{\text {Strong }}$ (or strong) and far $\bar{Z}_{\text {Weak }}$ (or weak) interactions as

$$
\overline{\boldsymbol{Z}}=\overline{\boldsymbol{Z}}_{\text {Strong }}+\overline{\boldsymbol{Z}}_{\text {Weak }},
$$

where the elements of the strong interactions are calculated from equation (1) (without approximation) and those of the weak interactions from the equation derived in appendix A. Equation (1) shows that $Z_{m, n}$ requires the calculation of two two-fold numerical integrations over the surfaces of the triangles $\mathrm{T}_{p}$ and $\mathrm{T}_{q}$. This is done from two-fold GaussLegendre integrations. In this paper, the weak interactions are derived from a closed-form expression addressed in appendix A. The singularity, which occurs for $D_{p, q}=0$, is computed from the work published by [19].

Then, calculating the sum over the 4 facets of edges $(m, n)$, appendix A shows that an element of the weak interaction is

$$
\begin{aligned}
\tilde{Z}_{m, n} \approx & \sum_{p=1}^{p=3} G^{(p)}\left(\boldsymbol{R}_{m}-\boldsymbol{R}_{n}\right) W^{(p)}\left(\boldsymbol{R}_{m}, \boldsymbol{R}_{n}\right) \\
= & \sum_{p=1}^{p=3} G^{(p),(0)}\left(r_{m, n}\right) \sum_{q=0}^{q=Q} W^{(p)}\left(\boldsymbol{R}_{m}, \boldsymbol{R}_{n}\right) \\
& \times\left(z_{m}-z_{n}\right)^{2 q} A^{(p),(q)}\left(r_{m, n}\right),
\end{aligned}
$$

where $\boldsymbol{R}_{m, n}=\boldsymbol{r}_{m, n}+\left(z_{m}-z_{n}\right) \hat{\boldsymbol{z}}$ and $G_{m, n}^{(p),(0)}=$ $\left.G_{m, n}^{(p)}\right|_{z_{n}-z_{m}=0}$. The above equation shows that the matrix $\tilde{\boldsymbol{Z}}_{m, n}$ is expressed as the sum over three matrices $\left\{\overline{\boldsymbol{G}}^{(p)}\right\}$ that depend only on $\boldsymbol{R}_{m}-\boldsymbol{R}_{n}$ weighted by polynomial functions $\left\{W^{(p)}\right\}$ that depend on the source edge $\boldsymbol{R}_{n}$ (its middle) and observation edge $\boldsymbol{R}_{m}$ (its middle). In addition, the element $G_{m, n}^{(p)}$ can be expanded over $z=z_{m}-z_{n}$ near 0 , where $z$ is the elevation difference between the edge middles $m$ and $n$. It is important to underline that $G_{m, n}^{(p),(0)}$ and $A_{m, n}^{(p),(q)}$ (weighting of the Taylor series expansion) depend only on $r_{m, n}=\sqrt{\left(x_{m}-x_{n}\right)^{2}+\left(y_{m}-y_{n}\right)^{2}}$. The functions $W_{m, n}^{(p)}$, $G_{m, n}^{(p),(0)}$ and $A_{m, n}^{(p),(q)}$ are expressed from equations (A15), (A17), (A20) $(Q=1)$ and (A21) $(Q=2)$.

The appendix also shows that equation (6) is valid if the strong interaction distance (equations (A6) and (A22)) satisfies

$$
r_{\text {Strong }}>\max \left(\frac{\max \left(L_{m}, \Delta z\right)^{2} n_{0}}{2 \lambda}, \max (\Delta z) \sqrt{\frac{n_{0}}{2}}\right),
$$

where $n_{0}=20, \Delta z=\max (z)-\min (z)$ on the distance $r_{\text {Strong }}$ and $L_{m}$ the $m$ edge length.

If the $\left(x_{m}, y_{m}\right)$ (or $\left(x_{n}, y_{n}\right)$ ) Cartesian grid is uniform, then the matrix associated to the element $G_{m, n}^{(p),(0)} \times A_{m, n}^{(p),(q)}=$ $B_{m, n}^{(p),(q)}$ is Toeplitz. In addition, since $W_{m, n}^{(p)}=\sum_{s=1}^{s=S} f_{m}^{(s)} g_{n}^{(s)}$ is a polynomial function of $\boldsymbol{R}_{n}$ and $\boldsymbol{R}_{m}$, equation (6) can be 
written as

$$
\begin{aligned}
& \tilde{Z}_{m, n} \approx \sum_{p=1}^{p=3} \sum_{q=0}^{q=Q} \sum_{r=0}^{R=2 q} W_{m, n}^{(p)} B_{m, n}^{(p),(q)}\left(z_{m}\right)^{2 q-r}\left(-z_{n}\right)^{r} C_{2 q}^{r} \\
&= \sum_{p=1}^{p=3} \sum_{s=1}^{s=S} \sum_{q=0}^{q=Q} \sum_{r=0}^{R=2 q} C_{2 q}^{r}\left[\left(z_{m}\right)^{2 q-r} f_{m}^{(s)}\right] B_{m, n}^{(p),(q)} \times \\
& {\left[\left(-z_{n}\right)^{r} g_{n}^{(s)}\right], }
\end{aligned}
$$

where $B_{m, n}^{(p),(q)}=G^{(p),(0)}\left(r_{m, n}\right) A^{(p),(q)}\left(r_{m, n}\right), C_{2 q}^{r}=$ $(2 q) ! /[r !(2 q-r) !]$ (binomial coefficient). The above equation is in a form such that the source point $n$ is on the right side of $B_{m, n}^{(p),(q)}$ while the observation point $m$ is located on the left side of $B_{m, n}^{(p),(q)}$. Appendix A shows that $S=3+6+36=45$ and gives the expression of $\left\{g_{n}^{(s)}, f_{m}^{(s)}\right\}$ for $s=\{1,2,3\}$, corresponding to $p=1$. The terms defined for $s>3$ are obtained in a similar way.

The integer $S$ corresponds to the expansion order that decomposes any function as a sum of $S$ terms that depend only on the $(m, n)$ product. For example, the dot product $\boldsymbol{\rho}_{m} \cdot \boldsymbol{\rho}_{n}=\rho_{m, x} \rho_{n, x}+\rho_{m, y} \rho_{n, y}+\rho_{m, z} \rho_{n, z}$, where the subscripts $(x, y, z)$ denote the vector components. Then $S=3$. This way is applied in Eq. (A $\underset{\sim}{2}$ ).

Then, the matrix product $\boldsymbol{O} \tilde{\bar{Z}} \boldsymbol{S}$ can be computed from $3 \times$ $45 \times[Q(Q+2)+1]$ FFTs, where $\boldsymbol{O}$ and $\boldsymbol{S}$ are any observation and source vectors, respectively.

\section{TOEPLITZ SUB-MATRICES}

As shown in Figure 1, the $\left(x_{m}, y_{m}\right)$ (or $\left(x_{n}, y_{n}\right)$ ) Cartesian grid over the center of the edges is not uniform, but the grid can be uniform by blocks. To this end, as shown in Fig. 1, the edges are sorted in ascending order going from left to right and from bottom to top. In what follows, the edges of $y$ values equal to $\{-0.15,-0.5,0.5,0.15\} \lambda$ are named odd rows whereas those of $y$ values equal to $\{-0.1,0,0.1\} \lambda$ are named even rows.

$\begin{array}{ccccc}\text { Let } f \text { be any } & \text { function of } & r_{m, n} & = \\ \sqrt{\left(x_{n}-x_{m}\right)+\left(y_{n}-y_{m}\right)^{2}} & \text { (planar surface) and } Z_{m, n}\end{array}$ an element of the matrix $\bar{Z}$ associated to the function $f\left(r_{m, n}\right)$. Then, as shown in Figure $2, \bar{Z}$ is Toeplitz by blocks by considering 4 cases:

1) The purple color represents the Toeplitz sub-matrices between the edges of odd rows.

2) The blue color represents the Toeplitz sub-matrices between the edges of even rows.

3) The green color represents the Toeplitz sub-matrices between the edges of odd rows and even rows, for which the edge numbers are odd.

4) The yellow color represents the Toeplitz sub-matrices between the edges of odd rows and even rows, for which the edge numbers are even.

In addition, for two rows separated by the same $y$ distance, the matrix is the same. For example, in Fig. 2, the sub-matrix of edges $m=n=\{1,2,3,4,5,6,7\}$ is the same as that obtained for $m=n=\{12,13,14,15,16,17,18\}$. For cases 1, 2 and 3, the sub-matrices are also symmetric, which implies

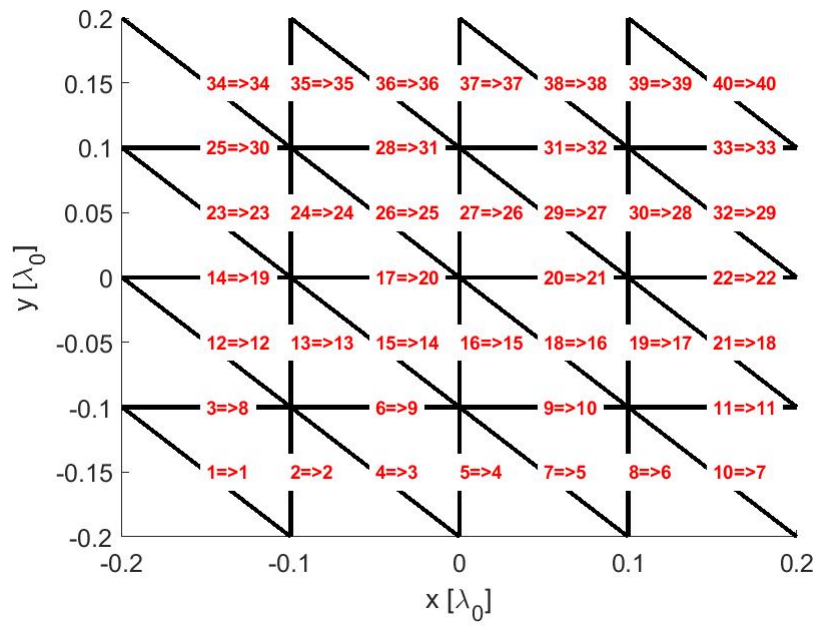

Fig. 1. Sort of the edges. A square plate of area $L_{x} \times L_{y}=(0.4 \lambda)^{2}$ is considered and the sampling steps with respect to the $x$ and $y$ directions are $\Delta x=\Delta y=0.1 \lambda$. The label $m \Rightarrow m^{\prime}$ (or $n \Rightarrow n^{\prime}$ ) means that the first number $m$ indicates the original edge number and the second number $m^{\prime}$ gives the new edge number. The integer $m^{\prime}$ sorts the edges in ascending order going from left to right and from bottom to top.

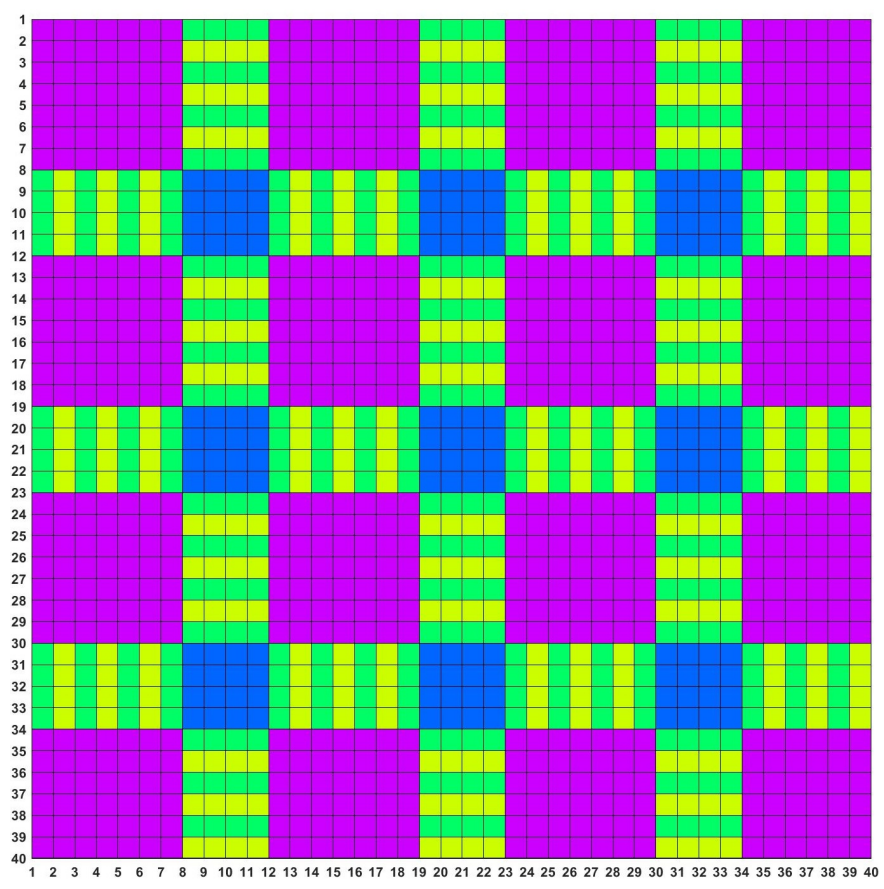

Fig. 2. Structure of the impedance matrix represented by Toeplitz submatrices.The four cases are represented by four colors and the geometry is shown in Fig. 1.

that only the first row is calculated, whereas for case 4 , the first row and column are computed.

For each case, Table I lists the length of the rows or columns of the sub-matrices and their number. $N_{x, y}$ is the number of vertices on a uniform grid of step $\Delta x$ and $\Delta_{y}$, respectively. For instance, in Fig. $1, \Delta x=\Delta y=0.1 \lambda, N_{x}=N_{y}=5$.

The total number of elements is then $N_{\text {Toep }}=6 N_{x} N_{y}$ $10 N_{x}-8 N_{y}+13$, while the number of edges is $N_{\text {Edge }}=$

\section{ACCEPTED MANUSCRIPT}


TABLE I

FOR EACH CASE, NUMBER AND LENGTH OF THE ROWS OR COLUMNS OF THE SUB-MATRICES. FOR CASE 4, THE LAST LINE CORRESPONDS TO THE COLUMNS.

\begin{tabular}{ccc}
\hline Case & Number & Length \\
\hline 1 & $N_{y}-1$ & $2 N_{x}-3$ \\
2 & $N_{y}-2$ & $N_{x}-1$ \\
3 & $N_{y}-2$ & $N_{x}-1$ \\
4 & $N_{y}-2$ & $N_{x}-1$ \\
4 & $N_{y}-2$ & $N_{x}-2$ \\
\hline
\end{tabular}

$3 N_{x} N_{y}-4\left(N_{x}+N_{y}\right)+5$. For $N_{x} \gg 1$ and $N_{y} \gg 1, N_{\text {Toep }} \approx$ $2 N_{\text {Edge }}$. For the weak interactions and for a planar surface, this means that instead of calculating $N_{\text {Edge }}^{2}-N_{\text {Strong }}$ elements, only $2 N_{\text {Edge }}$ elements are computed, where $N_{\text {Strong }}$ is the number of edge pairs in near field. In other words, for $N_{\text {Edge }}^{2} \gg N_{\text {Strong }}$, the compression rate is of the order of $1-2 / N_{\text {Edge }}$.

In addition, from the previous subsection and Table I, for $N_{x, y} \gg 1$, the complexity of the matrix-vector product $\bar{Z} \boldsymbol{X}=$ $\left(\overline{\boldsymbol{Z}}_{\text {Strong }}+\overline{\boldsymbol{Z}}_{\text {Weak }}\right) \boldsymbol{X}$ is

$$
\begin{aligned}
C_{\mathrm{MVP}} & =135 \times[Q(Q+2)+1] \times 4 \times N_{y} \log _{2} N_{x}+N_{\text {Strong }} \\
& =540[Q(Q+2)+1] N_{y} \log _{2} N_{x}+N_{\text {Strong }} \\
& \approx \alpha \sqrt{N_{\text {Edge }}} \log _{2} N_{\text {Edge }}+N_{\text {Strong }}
\end{aligned}
$$

where $\alpha=270[Q(Q+2)+1] / \sqrt{3}, N_{\text {Edge }} \approx 3 N_{x} N_{y}=3 N_{x}^{2}$ with $N_{y}=N_{x}$. For $r_{\text {Strong }}=0, N_{\text {Strong }}=N_{\text {Edge }}^{2}\left(\overline{\boldsymbol{Z}}_{\text {Weak }}=\overline{\mathbf{0}}\right)$. Then, in comparison to a conventional matrix-vector product of complexity $N_{\text {Edge }}^{2}$, the use of FFTs is efficient if

$$
\eta_{\text {MVP }}=\frac{\alpha \log _{2} N_{\text {Edge }}}{N_{\text {Edge }}^{3 / 2}}+\frac{N_{\text {Strong }}}{N_{\text {Edge }}^{2}} \ll 1 .
$$

\section{RESOLUTION OF THE LINEAR SYSTEM}

The final step is to efficiently solve the linear system $\bar{Z} \boldsymbol{X}=$ $\boldsymbol{b}$. For large problems, the conventional LU decomposition cannot be used and iterative schemes are preferred. Usually, the conjugate gradient algorithm and their improved versions can be good candidates, but for the EFIE, their convergence order is very large (for the scenarii presented in the next section, the order exceeds 300 ). In this paper, we propose to use $\overline{\boldsymbol{Z}}_{\text {Strong }}$ as a preconditioning matrix $\overline{\boldsymbol{M}}_{c}=\overline{\boldsymbol{Z}}_{\text {Strong }}^{-1} \overline{\boldsymbol{Z}}_{\text {Weak }}$ and next, a Taylor series expansion is applied on the resulting characteristics matrix to account for the weak interactions through $\bar{Z}_{\text {Weak }}$. Then

$$
\begin{aligned}
\boldsymbol{X} & =\left(\overline{\boldsymbol{Z}}_{\text {Strong }}+\overline{\boldsymbol{Z}}_{\text {Weak }}\right)^{-1} \boldsymbol{b} \\
& =\left[\overline{\boldsymbol{Z}}_{\text {Strong }}\left(\overline{\boldsymbol{I}}+\overline{\boldsymbol{Z}}_{\text {Strong }}^{-1} \overline{\boldsymbol{Z}}_{\text {Weak }}\right)\right]^{-1} \boldsymbol{b} \\
& =\left(\overline{\boldsymbol{I}}+\overline{\boldsymbol{M}}_{c}\right)^{-1} \overline{\boldsymbol{Z}}_{\text {Strong }}^{-1} \boldsymbol{b} \\
& \approx \sum_{k=0}^{K}\left(-\overline{\boldsymbol{M}}_{c}\right)^{k} \overline{\boldsymbol{Z}}_{\text {Strong }}^{-1} \boldsymbol{b}=\sum_{k=0}^{K} \overline{\boldsymbol{Y}}^{(k)},
\end{aligned}
$$

in which

$$
\left\{\begin{array}{ll}
\overline{\boldsymbol{Y}}^{(0)}=\overline{\boldsymbol{Z}}_{\text {Strong }}^{-1} \boldsymbol{b} & k=0 \\
\overline{\boldsymbol{Y}}^{(k+1)}=-\overline{\boldsymbol{M}}_{c} \overline{\boldsymbol{Y}}^{(k)}=-\overline{\boldsymbol{Z}}_{\text {Strong }}^{-1}\left(\overline{\boldsymbol{Z}}_{\text {Weak }} \overline{\boldsymbol{Y}}^{(k)}\right) & k>0
\end{array} .\right.
$$

This algorithm converges if the spectral radius (largest modulus of its eigenvalues) of $\overline{\boldsymbol{M}}_{c}$ is strictly smaller than one. This implies that the strong distance must be not too small. For large problems, this value is not calculated because it is very time consuming. Since $\bar{Z}_{\text {Strong }}$ is a sparse matrix, its LU decomposition matrices is also sparse, but this operation can be time consuming. To overcome this issue, the LSQR [18] (least squares QR) algorithm is applied because it is efficient for a sparse matrix and more efficient than a gradient conjugatebased method.

From equation (12), the complexity of the I-LSQR (I as iterative) algorithm is

$$
C_{\mathrm{I}-\mathrm{LSQR}}=C_{\mathrm{LSQR}}+K_{\mathrm{I}-\mathrm{LSQR}}\left(C_{\mathrm{LSQR}}+C_{\mathrm{MVP}}\right)
$$

where $C_{\mathrm{LSQR}}$ is the complexity of the LSQR algorithm and $C_{\mathrm{MVP}}$ is expressed from equation (9). The convergence order $K_{\mathrm{I}-\mathrm{LSQR}}=k$ is obtained when the relative residual error (RRE) satisfies

$$
\operatorname{RRE}=\frac{\operatorname{norm}\left(\boldsymbol{X}^{(k+1)}-\boldsymbol{X}^{(k)}\right)}{\operatorname{norm}\left(\boldsymbol{X}^{(k+1)}\right)}<\epsilon_{\mathrm{I}-\mathrm{LSQR}},
$$

where $\boldsymbol{X}^{(K)}=\sum_{k=0}^{k=K} \boldsymbol{Y}^{(k)}$ and $\epsilon_{\mathrm{I}-\mathrm{LSQR}}$ is the threshold of I-LSQR. Typically, $\epsilon_{\mathrm{I}-\mathrm{LSQR}}=10^{-2}$ and $\epsilon_{\mathrm{LSQR}}=5 \times 10^{-4}$ equals the LSQR threshold.

\section{NUMERICAL RESULTS}

The wavelength in free space $\lambda$ is $1 \mathrm{~m}$ and the polarization is $\theta \theta$.

\section{A. Paraboloid surface}

First, a paraboloid surface of equation $z(x, y)=2 a\left(x^{2}+\right.$ $\left.y^{2}\right) / L_{x}^{2}$ is considered and it is shown in Figure 3. In the $(O x, O y)$ plane, the surface area is $L_{x} L_{y}$, where $\left\{L_{x, y}\right\}$ are the surface lengths with respect to the $x$ and $y$ directions. In addition, the center of the surface is the point $O$ of coordinates $(0,0)$. For $x=L_{x} / 2$ and $y=L_{y} / 2=L_{x} / 2$, $z=a=\max (z)$. In Figure 3, the number of edges is $N_{\text {Edge }}=42,960$, the sampling steps with respect to the $x$ and $y$ directions are $\Delta x=\Delta y=0.1 \lambda$ and $a=\lambda / 2$.

Fig 4 plots the bistatic RCS in $\mathrm{dBm}^{2}$ versus the scattering angle $\theta_{\text {sca. }}$. To better highlight the differences, in the lower subfigure, the ratio $\mathrm{RCS}_{\mathrm{I}-\mathrm{LSQR}} / \mathrm{RCS}_{\mathrm{LU}}$ is plotted in $\mathrm{dB}$ scale, where $\mathrm{RCS}_{\mathrm{LU}}$ is the RCS computed from a $\mathrm{LU}$ decomposition of the impedance matrix. The incidence angles are $\theta_{\text {inc }}=\pi / 6$ and $\phi_{\text {inc }}=0$, and $\phi_{\text {sca }}=0$ is the azimuthal scattering angle. The illuminated object is a paraboloid surface, as shown in Figure 3. In the legend, "LU: $\left(t_{\mathrm{LU}, 1}, t_{\mathrm{LU}, 2}\right) s$ " and " $u_{\text {Strong }}, \mathrm{I}-\mathrm{LSQR}-\left(Q, K_{\mathrm{I}-\mathrm{LSQR}}, N_{\mathrm{LSQR}}\right)$ : $\left(t_{\mathrm{I}-\mathrm{LSQR}, 1}, t_{\mathrm{I}-\mathrm{LSQR}, 2}, t_{\mathrm{I}-\mathrm{LSQR}, 3}\right) s, \mathrm{RRE}$ ” mean

- $t_{\mathrm{LU}, 1}$ : Computing time to fill the impedance matrix, in seconds.

- $t_{\mathrm{LU}, 2}$ : Computing time to solve the linear system from LU, in seconds.

- $u_{\text {Strong }}=r_{\text {Strong }} / \lambda$ : Distance of the strong interactions normalized by $\lambda$. 


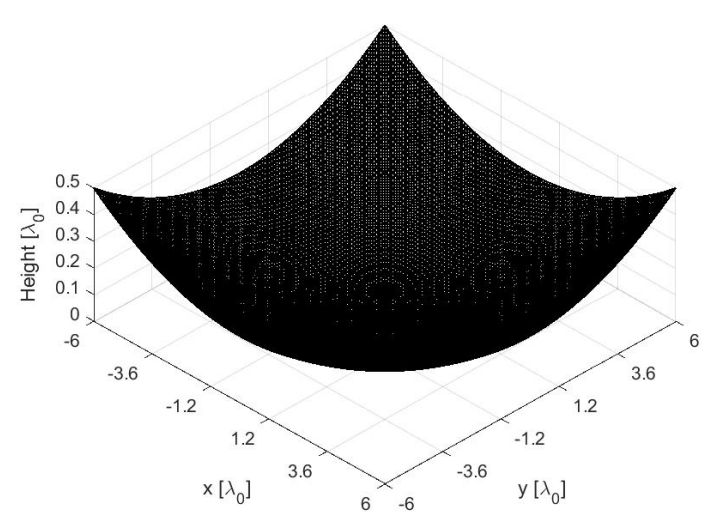

Fig. 3. Paraboloid surface with $L_{x}=L_{y}=12 \lambda . N_{\text {Edge }}=42,960$, $\Delta x=\Delta y=0.1 \lambda$ and $a=\lambda / 2$.

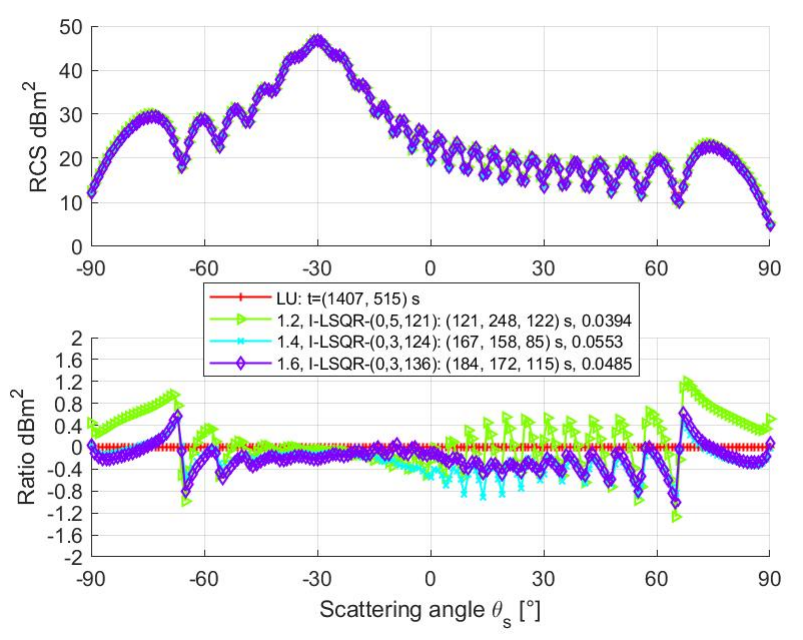

Fig. 4. Top: Bistatic RCS in $\mathrm{dBm}^{2}$ versus the scattering angle $\theta_{\text {sca. }}$. Bottom: Ratio $\mathrm{RCS}_{\mathrm{I}-\mathrm{LSQR}} / \mathrm{RCS}_{\mathrm{LU}}$ in dB scale. $Q=0, r_{\text {Strong }}=\{1.2,1.4,1.6\} \lambda$, $\theta_{\text {inc }}=\pi / 6, \phi_{\text {inc }}=0$ and $\phi_{\text {sca }}=0$. The illuminated object is a paraboloid surface, as shown in Figure 3.

- $K_{\mathrm{I}-\mathrm{LSQR}}$ : Convergence order of I-LSQR.

- $N_{\mathrm{LSQR}}$ : Mean convergence order of LSQR $\left(\epsilon_{\mathrm{LSQR}}=5 \times\right.$ $10^{-4}$ ).

- $Q$ : Order of the Taylor series expansion over $z$.

- $t_{\mathrm{I}-\mathrm{LSQR}, 1}$ : Computing time to fill the impedance matrix of the strong interactions, in seconds.

- $t_{\mathrm{I}-\mathrm{LSQR}, 2}$ : Computing time to compute the matrix-vector product $\overline{\boldsymbol{Z}}_{\text {Weak }} \overline{\boldsymbol{Y}}^{(k)}=\boldsymbol{v}$, in seconds.

- $t_{\mathrm{I}-\mathrm{LSQR}, 3}$ : Computing time to solve the linear system $\overline{\boldsymbol{Z}}_{\text {Strong }}^{-1} \boldsymbol{v}$ from LSQR $\left(\epsilon_{\mathrm{LSQR}}=5 \times 10^{-4}\right)$, in seconds.

- RRE: Relative residual error obtained at the convergence order $K_{\mathrm{I}-\mathrm{LSQR}}\left(\epsilon_{\mathrm{I}-\mathrm{LSQR}}=10^{-2}\right)$.

The total computing time is then $t_{\mathrm{LU}, 1}+t_{\mathrm{LU}, 2}$ for $\mathrm{LU}$ and $t_{\mathrm{I}-\mathrm{LSQR}, 1}+t_{\mathrm{I}-\mathrm{LSQR}, 2}+t_{\mathrm{I}-\mathrm{LSQR}, 3}$ for I-LSQR.

As we can see in Fig. 4, the results match well with those obtained from LU. As $r_{\text {Strong }}$ increases, the results better match and the order of convergence, $K_{\mathrm{I}-\mathrm{LSQR}}$, decreases from 5 to 3 and the filling time $t_{\mathrm{I}-\mathrm{LSQR}, 1}$ increases slightly. By

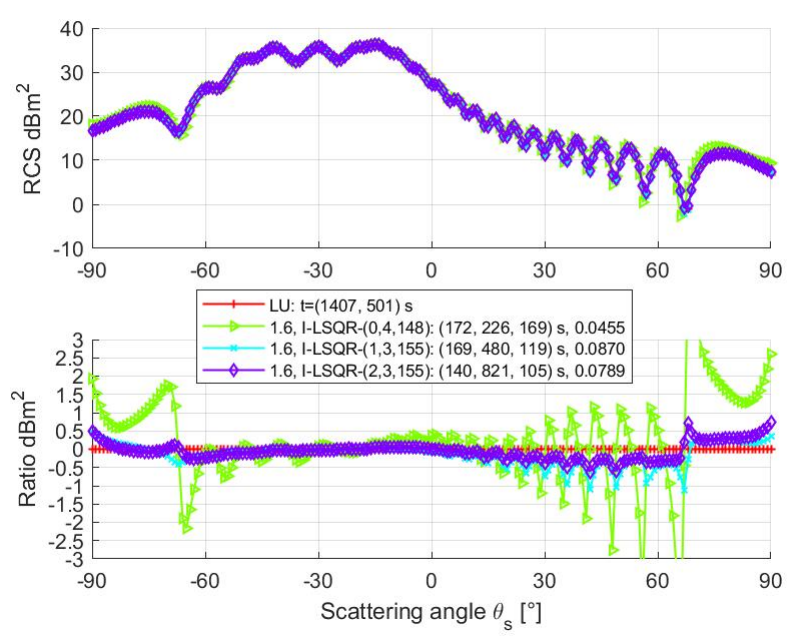

Fig. 5. Same variations as in Fig. 4 but $a=1.5 \lambda, r_{\text {Strong }}=1.6 \lambda$ and $Q=\{0,1,2\}$.

calculating only $\bar{Z}_{\text {Strong, }}$, the memory requirement is divided by 20 in comparison to $\mathrm{LU}$, which needs to calculate all the elements of $\bar{Z}$. Compared to the filling computation time of $\mathrm{LU}$, the gain is of the order of 9. On the other hand, the time $t_{\mathrm{I}-\mathrm{LQR}, 2}+t_{\mathrm{I}-\mathrm{LSQR}, 3}$ to solve the linear system by I-LSQR is slightly smaller than that of $\mathrm{LU}, t_{\mathrm{LU}, 2}$.

From equation (10), $\eta_{\mathrm{MVP}} \approx 0.05$, with $r_{\text {Strong }}=1.6 \lambda$. This means that the matrix-vector product computed from $N_{\mathrm{FFT}}$ FFTs should be $1 / \eta_{\mathrm{MVP}} \approx 20$ faster than the one computed in a conventional manner. In practice, it is not the case. The FFTs (and IFFTs) are computed from the fft and ifft MatLab functions in a matrix manner to accelerate their calculation. Nevertheless, loops are required and the reshape MatLab function is also applied, which increases the computing time. If this step was programmed in $\mathrm{C}$, the computing time would be smaller. We can also note that this time $t_{\mathrm{I}-\mathrm{LSQR}, 2}$ is larger than $t_{\mathrm{I}-\mathrm{LSQR}, 3}$, allocated to solve the linear system by LSQR, also computed in MatLab but by calling $\mathrm{C}$ functions.

However, both computing times $\left(t_{\mathrm{I}-\mathrm{LSQR}, 2}, t_{\mathrm{I}-\mathrm{LSQR}, 3}\right)$ are not comparable and $t_{\mathrm{I}-\mathrm{LSQR}, 2}$ would be much smaller if the matrixvector product was made in C. In addition, a parallelization would make it possible to significantly to decrease this computation time. With MatLab, the LU inversion is optimized and parallelized.

Fig. 5 plots the same variations as in Fig. 4 but $a=1.5 \lambda$, $r_{\text {Strong }}=1.6 \lambda$ and $Q=\{0,1,2\}$. As expected, as $Q$ increases, the results better match with those obtained from LU, and the results computed for $Q=1$ and $Q=2$ are nearly the same.

Equation (7) gives the lowest value of the strong interaction distance, for which the derivation of the weak interaction matrix is valid at order zero $(Q=0)$. In polar coordinates $z(x, y)=h(r)=2 a r^{2} / L_{x}^{2}$ and $d h / d r=4 a r / L_{x}^{2}$. Then, $\max (\Delta z) \approx 4 a \max (r) r_{\text {Strong }} / L_{x}^{2}=2 a r_{\text {Strong }} / L_{x}=0.4 \lambda$ in Fig. 5. From equation (7), $r_{\text {Strong }}>\max (0.22,1.60,126) \lambda=$ $1.6 \lambda=r_{\text {Strong,min }}$ with $n_{0}=20$. This explains in Fig. 5 why the results for $Q=0$ deviate from those obtained from LU for low values of the RCS since $r_{\text {Strong }}=r_{\text {Strong,min }}$.

\section{ACCEPTED MANUSCRIPT}




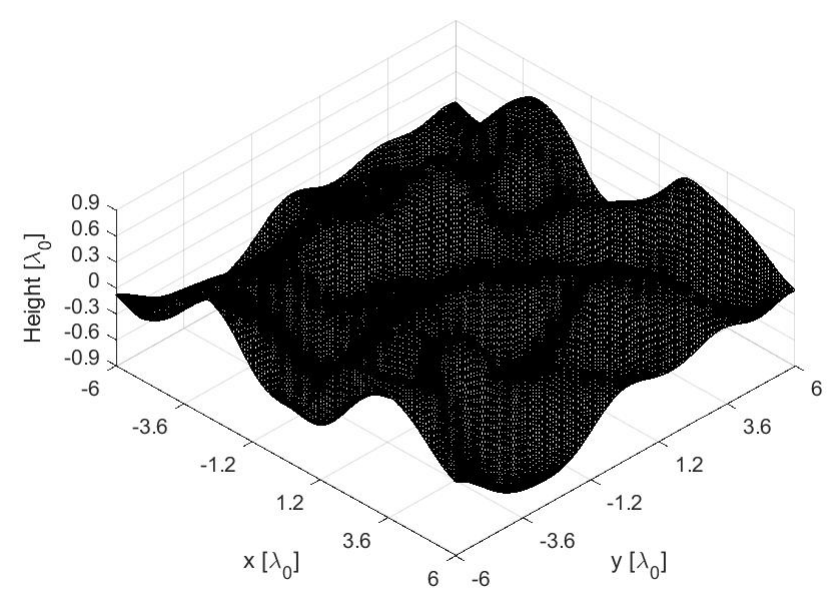

Fig. 6. Surface height versus the coordinates $x$ and $y . L_{x} \times L_{y}=144 \lambda^{2}$, $\sigma_{z}=0.3 \lambda$ and $L_{c, x}=L_{c, y}=1.5 \lambda$. The number of edges is $N_{\text {Edge }}=$ $42,960$.
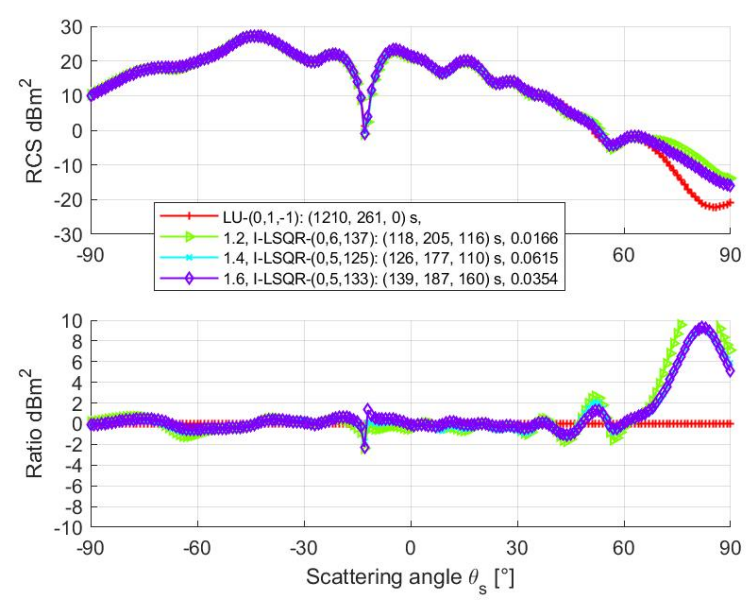

Fig. 7. Top: Bistatic RCS in $\mathrm{dBm}^{2}$ versus the scattering angle $\theta_{\text {sca. }}$. Bottom: Ratio $\mathrm{RCS}_{\mathrm{I}-\mathrm{LSQR}} / \mathrm{RCS}_{\mathrm{LU}}$ in $\mathrm{dB}$ scale. $Q=0, r_{\mathrm{Strong}}=\{1.2,1.4,1.6\} \lambda$, $\theta_{\text {inc }}=\pi / 6, \phi_{\text {inc }}=0$ and $\phi_{\text {sca }}=0$. The illuminated object is a rough surface, which is plotted in Figure 6.

The order $Q=1$ makes it possible to significantly decrease this deviation, and the contribution of the second order is negligible.

\section{B. Rough surface}

To strongly attenuate the edge diffractions by the surface, the well-known incident tapered wave published by Braunish et al. [20] is applied (at the order two) with tapering parameter $g=L_{x} / 4$ (surface of area $A_{0}=L_{x}^{2}$ ). The length $g$ controls the extent of the incident beam that illuminates the surface.

Fig. 6 plots a random rough surface of Gaussian height distribution and Gaussian height autocorrelation function. The surface correlation lengths with respect to the $x$ and $y$ directions are $L_{c, x}=L_{c, y}=1.5 \lambda$, the surface height standard deviation is $\sigma_{z}=0.3 \lambda$ and the surface area is $L_{x} \times L_{y}=144 \lambda^{2}$.

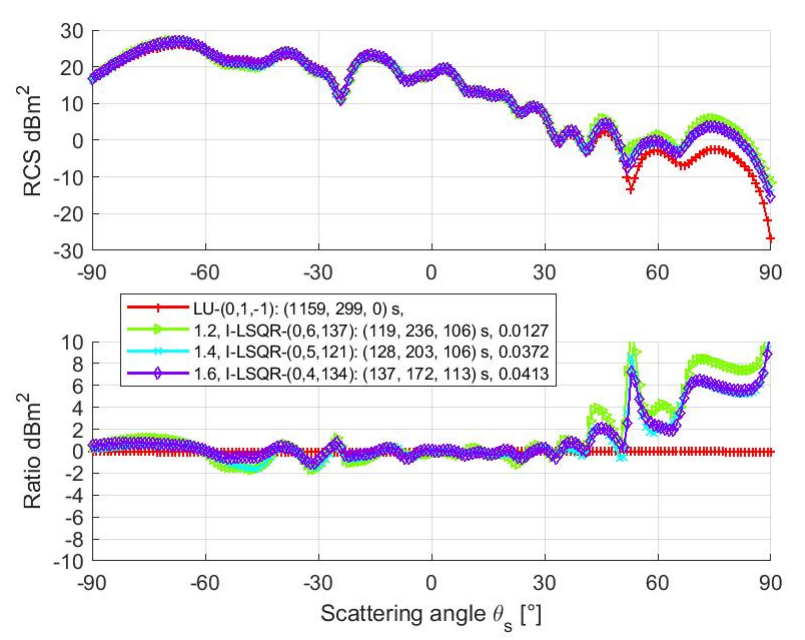

Fig. 8. Same variations as in Fig. 7 but the incidence angle $\theta_{\text {inc }}=\pi / 4$.
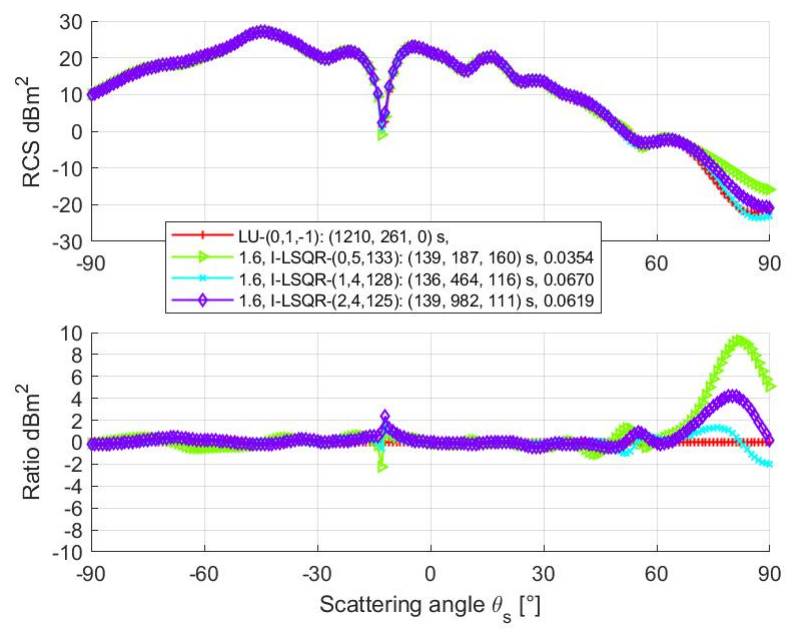

Fig. 9. Same variations as in Fig. 7 but $r_{\text {Strong }}=1.6 \lambda$ and $Q=\{0,1,2\}$.

Fig. 7 plots the bistatic RCS in $\mathrm{dBm}^{2}$ versus the scattering angle $\theta_{\text {sca }}$. At the bottom, the ratio $\mathrm{RCS}_{\mathrm{I}-\mathrm{LSQR}} / \mathrm{RCS}_{\mathrm{LU}}$ is plotted in $\mathrm{dB}$ scale. $Q=0, r_{\text {Strong }}=\{1.2,1.4,1.6\} \lambda$, $\theta_{\text {inc }}=\pi / 6, \phi_{\text {inc }}=0$ and $\phi_{\text {sca }}=0$. The illuminated object is a rough surface, which is plotted in Figure 6. For high values of RCS, the results match well with those obtained from LU. From equation (7) and by taking $\Delta z \approx \sqrt{2} \sigma_{z}$, $r_{\text {Strong }}>\max (0.34,1.80,1.34) \lambda=1.8 \lambda$. In Fig. 7, this explains why differences with LU appear for low values of RCS.

Fig. 8 plots the same variations as in Fig. 7 but the incidence angle $\theta_{\text {inc }}=\pi / 4$ (instead of $\theta_{\text {inc }}=\pi / 6$ ). Like in Fig. 7, a deviation occurs from incidence angles larger than 30-45 degrees and the proposed method gives similar performances.

Fig. 9 plots the same variations as in Fig. 7 but $r_{\text {Strong }}=$ $1.6 \lambda$ and $Q=\{0,1,2\}$. For $Q=1$, the results match well with those obtained from LU. Fig. 9 also shows that it is not relevant to calculate the order $Q=2$.

Fig. 10 plots the same variations as in Fig. 9 but in cross

\section{ACCEPTED MANUSCRIPT}



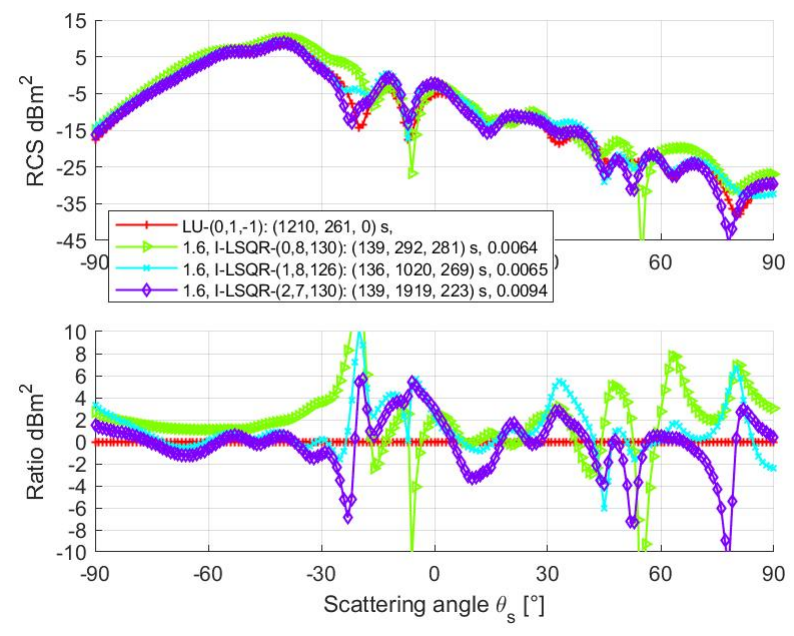

Fig. 10. Same variations as in Fig. 9 but in cross polarization $(\theta \phi$ or VH).

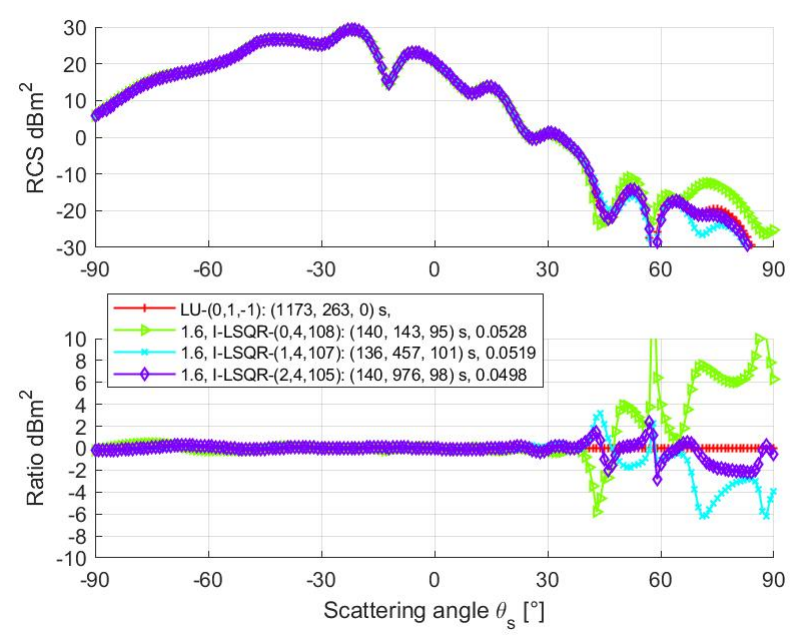

Fig. 11. Same variations as in Fig. 9 but $L_{c, x}=L_{c, y}=2 \lambda$ (instead of $\left.L_{c, x}=L_{c, y}=1.5 \lambda\right)$.

polarization $(\theta \phi$ or $\mathrm{VH})$. The $\mathrm{VH}$ strengths are $20 \mathrm{~dB}$ lower than those obtained in $\mathrm{VV}$, which explains why the deviation with LU is larger than those shown in Fig. 9. Indeed, the cross polarization requires a better accuracy, since the levels are smaller.

Fig. 11 plots the same variations as in Fig. 9, but $L_{c, x}=$ $L_{c, y}=2 \lambda$ (instead of $L_{c, x}=L_{c, y}=1.5 \lambda$ ). As we can see, the results obtained for $Q=1$ match well with those obtained from LU. In comparison to Fig. 9, the total computing time is slightly smaller because $r_{\text {Strong }}$ remains unchanged and the mean convergence order $N_{I-\mathrm{LSQR}} \approx 117$ is smaller, whereas the convergence order $K_{\mathrm{LSQR}}=4$ remains constant.

Fig. 12 plots the same variations as in Fig. 9 but $r_{\text {Strong }}=$ $4.4 \lambda$ and $\sigma_{z}=0.5 \lambda$ (instead of $\sigma_{z}=0.3 \lambda$ ). The value $r_{\text {Strong }}=4.4 \lambda$ is chosen so that it satisfies criterion (7). As we can see, the results obtained for $Q=1$ match well with those computed from LU. In comparison to LU (and Fig. 9), the computing time is greater because $r_{\text {Strong }}$ is larger than
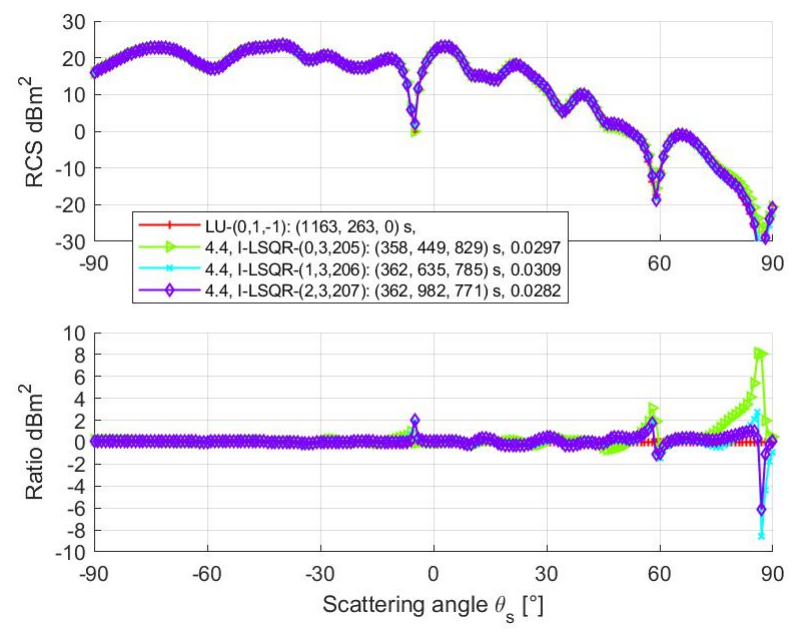

Fig. 12. Same variations as in Fig. 9 but $r_{\text {Strong }}=4.4 \lambda$ and $\sigma_{z}=0.5 \lambda$ (instead of $\sigma_{z}=0.3 \lambda$ ).

that used for $\sigma_{z}=0.3 \lambda$ (it is proportional to $\sigma_{z}^{2}$ ) and the mean convergence order $N_{I-\mathrm{LSQR}} \approx 206$ increases, whereas $K_{\mathrm{LSQR}}=3$ is smaller. As expected, as $\sigma_{z}$ increases, the proposed method is less efficient.

\section{CONCLUSION}

First, an acceleration to compute the impedance matrix, based on a far-field approximation, is addressed. Next, the impedance matrix is split into strong and weak interactions and this latter is compressed by expressing it from Toeplitz submatrices. Then, the linear system is efficiently solved from a bi-iterative scheme. For a given order, the LSQR (least squares $\mathrm{QR})$ algorithm is applied to solve the sparse linear system related to the strong interactions, while the matrix-vector products, related to the weak interactions, are accelerated by using FFTs.

For a paraboloid-shaped object, the numerical results show that the proposed method, named I-LSQR, is very efficient. The computation of the higher order (related to $\Delta z=$ $\max (z)-\min (z))$ makes it possible to obtain more accurate results with an increase of the computing time.

For a rough surface, the numerical results also show that I-LSQR is efficient, but it is more sensitive to $\Delta z \propto \sigma_{z}$. In addition, the method becomes less efficient as $\sigma_{z}$ increases, because the minimum strong interaction distance (7) increases. It is approximately expressed as $\left(\sqrt{2} \sigma_{z}\right)^{2} \times 20 /(2 \lambda)=20 \sigma_{z}^{2} / \lambda$.

\section{ACKNOWLEDGMENT}

The author thanks the anonymous reviewers for their useful comments. The author also thank Dr. Nicolas Pinel and Dr. Gildas Kubické for their suggestions that have contributed in improving the quality of this paper.

\section{APPENDIX A}

DERIVATION OF THE WEAK IMPEDANCE MATRIX ELEMENTS

For a pair of facets in far field from each other, using an updated Fraunhofer criterion, Bourlier [21], [22] showed that 


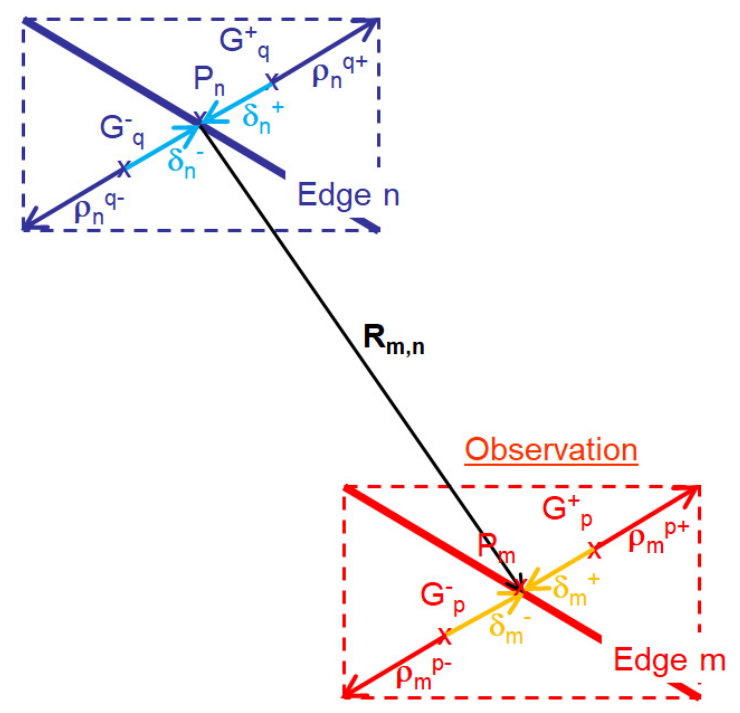

Fig. 13. For a planar surface, interaction of an edge pair $(m, n)$ and their two facets. The facets are assumed to be identical.

equation (1) can be simplified as

$$
\begin{aligned}
Z_{m, n} & \approx \frac{s_{p} s_{q} e^{-j k R_{p, q}}}{4 \pi A_{p} A_{q} R_{p, q} L_{m} L_{n}} \iint_{\mathrm{T}_{p}} \iint_{\mathrm{T}_{q}}\left(\boldsymbol{\rho}_{m}^{p} \cdot \boldsymbol{\rho}_{n}^{q}-\frac{1}{k^{2}}\right) \\
& \times e^{-j k \hat{\boldsymbol{R}}_{p, q} \cdot\left(\boldsymbol{\delta}_{p}-\boldsymbol{\delta}_{q}\right)} d R_{p} d R_{q},
\end{aligned}
$$

where $\boldsymbol{R}_{p, q}=\boldsymbol{G}_{p}-\boldsymbol{G}_{q}$ and $\boldsymbol{\delta}_{p, q}=\boldsymbol{M}_{p, q}-\boldsymbol{G}_{p, q}$, in which $\boldsymbol{G}_{p, q}$ is the gravity center of the facets $p$ and $q$, respectively, and $\boldsymbol{M}_{p, q}$ the integration point on the facets $p$ and $q$, respectively $\left(\boldsymbol{D}_{p, q}=\boldsymbol{M}_{p}-\boldsymbol{M}_{q}=\boldsymbol{R}_{p, q}+\boldsymbol{\delta}_{p}-\boldsymbol{\delta}_{q}\right)$. In addition, $\hat{\boldsymbol{R}}_{p, q}=\boldsymbol{R}_{p, q} / R_{p, q}$, where $R_{p, q}=\left\|\boldsymbol{R}_{p, q}\right\|$.

For the weak interactions, the integral can be evaluated from a single point chosen at the middle of the egde $m$ or $n, \boldsymbol{P}_{m, n}$. Equation (A1) becomes

$$
Z_{m, n} \approx \frac{s_{p} s_{q} e^{-j k R_{m, n}^{\prime}}}{4 \pi R_{m, n}^{\prime} L_{m} L_{n}}\left(\boldsymbol{\rho}_{m}^{p} \cdot \boldsymbol{\rho}_{n}^{q}-\frac{1}{k^{2}}\right),
$$

where

$$
R_{m, n}^{\prime}=R_{m, n}\left[1+\frac{\left(\boldsymbol{\delta}_{m}-\boldsymbol{\delta}_{n}\right) \cdot \boldsymbol{R}_{m, n}}{R_{m, n}^{2}}\right],
$$

and $\boldsymbol{\delta}_{p, q}=\boldsymbol{\delta}_{m, n}=\boldsymbol{P}_{m, n}-\boldsymbol{G}_{p, q}, \boldsymbol{R}_{p, q}=\boldsymbol{P}_{m}-\boldsymbol{P}_{n}=\boldsymbol{R}_{m, n}$. In comparison to equation (A2) at the denominator, $R_{p, q}$ is changed by $R_{p, q}^{\prime}$. Since $\left|\left(\boldsymbol{\delta}_{m}-\boldsymbol{\delta}_{n}\right) \cdot \boldsymbol{R}_{m, n}\right| / R_{m, n}^{2} \ll 1$, and by using the approximation $1 /(1+x) \approx 1-x \approx e^{-x}$ for $|x| \ll 1$, equation (A2) can be expressed as

$$
Z_{m, n} \approx \frac{s_{p} s_{q} e^{-j k R_{m, n}}}{4 \pi R_{m, n} L_{m} L_{n}}\left(\boldsymbol{\rho}_{m}^{p} \cdot \boldsymbol{\rho}_{n}^{q}-\frac{1}{k^{2}}\right) e^{j\left(\phi_{1}+\phi_{2}\right)},
$$

where

$$
\left\{\begin{array}{l}
\phi_{1}=-k \delta_{m} \hat{\boldsymbol{\delta}}_{m} \cdot \hat{\boldsymbol{R}}_{m, n}\left(1-\frac{j}{k R_{m, n}}\right) \\
\phi_{2}=+k \delta_{n} \hat{\boldsymbol{\delta}}_{n} \cdot \hat{\boldsymbol{R}}_{m, n}\left(1-\frac{j}{k R_{m, n}}\right)
\end{array} .\right.
$$

Equation (A4) is valid if $\Delta^{2} / 2 R_{m, n}<\lambda / n_{0}$ [21], where $n_{0}$ is an integer ranging from 10 to 20 and $\Delta=\max \left(\delta_{n}\right)+$ $\max \left(\delta_{m}\right) \approx \max \left(L_{m}\right)$. Typically, $n_{0}=20$. In other words, the distance of the strong interactions must satisfy

$$
R_{\text {Strong }}>\frac{n_{0} \max \left(L_{m}\right)^{2}}{2 \lambda} .
$$

Since an edge shares two facets, a pair of edges $(m, n)$ implies 4 facets. As shown in Fig. 13, assuming that the meshed triangles are identical, the sum over the facets of $Z_{m, n}$ can be made analytically. Since for a given edge, $\boldsymbol{\delta}_{m, n}^{-}=-\boldsymbol{\delta}_{m, n}^{+}$and $\rho_{m, n}^{p-, q-}=-\rho_{m, n}^{p+q+}$, the sum over the 4 triangles of $s_{p} s_{q} e^{j\left(\phi_{1}+\phi_{2}\right)}$ reduces to

$$
\begin{aligned}
& e^{j\left(\phi_{1}+\phi_{2}\right)}-e^{j\left(-\phi_{1}+\phi_{2}\right)}-e^{j\left(\phi_{1}-\phi_{2}\right)}+e^{-j\left(\phi_{1}+\phi_{2}\right)} \\
& =-4 \sin \phi_{1} \sin \phi_{2} .
\end{aligned}
$$

In the same way, the sum over the 4 triangles of $s_{p} s_{q} e^{j\left(\phi_{1}+\phi_{2}\right)} \boldsymbol{\rho}_{m}^{p} \cdot \boldsymbol{\rho}_{n}^{q} /\left(\boldsymbol{\rho}_{m}^{p+} \cdot \boldsymbol{\rho}_{n}^{q+}\right)$ reduces to

$$
\begin{aligned}
& e^{j\left(\phi_{1}+\phi_{2}\right)}+e^{j\left(-\phi_{1}+\phi_{2}\right)}+e^{j\left(\phi_{1}-\phi_{2}\right)}+e^{-j\left(\phi_{1}+\phi_{2}\right)} \\
& =4 \cos \phi_{1} \cos \phi_{2} .
\end{aligned}
$$

An element $\bar{Z}_{m, n}$ of the impedance matrix is then

$$
\begin{gathered}
\tilde{Z}_{m, n} \approx \frac{e^{-j k R_{m, n}}}{\pi R_{m, n} L_{m} L_{n}}\left(\boldsymbol{\rho}_{m}^{p+} \cdot \boldsymbol{\rho}_{n}^{q+} \cos \phi_{1} \cos \phi_{2}\right. \\
\left.+\frac{\sin \phi_{1} \sin \phi_{2}}{k^{2}}\right) .
\end{gathered}
$$

Applying the following identities [23]

$$
\left\{\begin{array}{l}
\cos (z \cos \theta)=\mathbf{J}_{0}(z)+2 \sum_{p=1}^{\infty} \mathbf{J}_{2 p}(z) \cos (2 p \theta) \\
\sin (z \cos \theta)=2 \sum_{p=1}^{\infty}(-1)^{p} \mathbf{J}_{2 p+1}(z) \cos [(2 p+1) \theta]
\end{array}\right.
$$

where $\mathbf{J}_{p}$ is the Bessel function of the first kind and order $p$, we have

$$
\left\{\begin{array}{l}
\cos \phi_{1} \cos \phi_{2} \approx \mathbf{J}_{0}\left(q_{m}\right) \mathbf{J}_{0}\left(q_{n}\right) \\
\sin \phi_{1} \sin \phi_{2} \approx-4 \mathbf{J}_{1}\left(q_{m}\right) \mathbf{J}_{1}\left(q_{n}\right) \cos \phi_{m} \cos \phi_{n}
\end{array},\right.
$$

where $\phi_{1}=-q_{m} \cos \phi_{m}$ and $\phi_{2}=q_{n} \cos \phi_{n}$. The use of Eq. (A5) leads to

$$
q_{m, n}=k \delta_{m, n}\left(1-\frac{j}{k R_{m, n}}\right), \quad \cos \phi_{m, n}=\hat{\boldsymbol{\delta}}_{m, n} \cdot \hat{\boldsymbol{R}}_{m, n} .
$$

In equation (A10), only the first term of the sum is kept, since $\left|q_{m, n}\right| \ll 1$.

In equation (A12), it is important to keep in mind that the extra term $j /\left(k R_{m, n}\right)$ comes from a Taylor series expansion up to the order one. Then, we can simplify equation (A11) as

$$
\begin{aligned}
& \cos \phi_{1} \cos \phi_{2} \approx \mathbf{J}_{0}\left(w_{m}\right) \mathbf{J}_{0}\left(w_{n}\right)+\frac{j}{k R_{m, n}} \times \\
& {\left[w_{m} \mathbf{J}_{1}\left(w_{m}\right) \mathbf{J}_{0}\left(w_{n}\right)+w_{n} \mathbf{J}_{1}\left(w_{n}\right) \mathbf{J}_{0}\left(w_{m}\right)\right]} \\
& \approx\left(1-\frac{w_{m}^{2}}{4}\right)\left(1-\frac{w_{n}^{2}}{4}\right)+\frac{j}{2 k R_{m, n}}\left(w_{m}^{2}+w_{n}^{2}\right),
\end{aligned}
$$


and

$$
\begin{aligned}
\frac{\sin \phi_{1} \sin \phi_{2}}{\cos \phi_{m} \cos \phi_{n}} & \approx-4 \mathbf{J}_{1}\left(w_{m}\right) \mathbf{J}_{1}\left(w_{n}\right)+\frac{2 j}{k R_{m, n}} \times \\
& \left\{w_{m} \mathbf{J}_{1}\left(w_{n}\right)\left[\mathbf{J}_{0}\left(w_{m}\right)-\mathbf{J}_{2}\left(w_{m}\right)\right]\right. \\
& \left.+w_{n} \mathbf{J}_{1}\left(w_{m}\right)\left[\mathbf{J}_{0}\left(w_{n}\right)-\mathbf{J}_{2}\left(w_{n}\right)\right]\right\} \\
& \approx-4 \mathbf{J}_{1}\left(w_{m}\right) \mathbf{J}_{1}\left(w_{n}\right)+\frac{2 j}{k R_{m, n}} \times \\
& {\left[w_{m} \mathbf{J}_{1}\left(w_{n}\right) \mathbf{J}_{0}\left(w_{m}\right)+w_{n} \mathbf{J}_{1}\left(w_{m}\right) \mathbf{J}_{0}\left(w_{n}\right)\right] } \\
& \approx-w_{m} w_{n}\left(1-\frac{2 j}{k R_{m, n}}\right) .
\end{aligned}
$$

where $w_{m, n}=k \delta_{m, n}$. In addition, since $\left|w_{m, n}\right| \ll 1$, $\mathbf{J}_{0}\left(w_{m, n}\right) \approx 1-w_{m, n}^{2} / 4$ and $\mathbf{J}_{1}\left(w_{m, n}\right) \approx w_{m, n} / 2$. These approximations makes the programming easier.

In conclusion, an element of the impedance can be approximated as

$$
\begin{aligned}
L_{m} L_{n} \tilde{Z}_{m, n} & \approx G_{m, n}^{(1)}\left(1-\frac{w_{m}^{2}}{4}\right)\left(1-\frac{w_{n}^{2}}{4}\right) \boldsymbol{\rho}_{m}^{p+} \cdot \boldsymbol{\rho}_{n}^{q+} \\
& +\left(w_{m}^{2}+w_{n}^{2}\right) G_{m, n}^{(2)} \boldsymbol{\rho}_{m}^{p+} \cdot \boldsymbol{\rho}_{n}^{q+} \\
& +w_{m} w_{n} G_{m, n}^{(3)}\left(\boldsymbol{R}_{m, n} \cdot \hat{\boldsymbol{\delta}}_{m}\right)\left(\boldsymbol{R}_{m, n} \cdot \hat{\boldsymbol{\delta}}_{n}\right)
\end{aligned}
$$

where

$$
\left\{\begin{array}{l}
w_{m, n}=k \delta_{m, n}=k\left\|\boldsymbol{\delta}_{m, n}\right\| \\
\boldsymbol{\delta}_{m, n}=\boldsymbol{P}_{m, n}-\boldsymbol{G}_{p+, q+} \\
\boldsymbol{R}_{m, n}=\boldsymbol{P}_{m}-\boldsymbol{P}_{n} \\
\boldsymbol{\rho}_{m, n}^{p+, q+}=\left(\boldsymbol{V}_{m, n}^{p+, q+}-\boldsymbol{G}_{p+, q+}\right) / 2
\end{array},\right.
$$

and

$$
\left\{\begin{array}{l}
G_{m, n}^{(1)}=\frac{e^{-j k R_{m, n}}}{\pi R_{m, n}} \\
G_{m, n}^{(2)}=G_{m, n}^{(1)} \frac{j}{2 k R_{m, n}} \\
G_{m, n}^{(3)}=-G_{m, n}^{(1)}\left(1-\frac{2 j}{k R_{m, n}}\right) \frac{1}{k^{2} R_{m, n}^{2}}
\end{array}\right.
$$

Since for a planar surface, $\boldsymbol{R}_{m, n}=\boldsymbol{r}_{m, n}=\left(x_{n}-x_{m}\right) \hat{\boldsymbol{x}}+$ $\left(y_{n}-y_{m}\right) \hat{\boldsymbol{y}}$ on a uniform grid, the matrix $\overline{\boldsymbol{Z}}_{m, n}^{(i)}$ associated to the element $G_{m, n}^{(i)}$ is Toeplitz. Then, the matrix product $\boldsymbol{O}_{m} \overline{\boldsymbol{Z}}_{m, n}^{(i)} \boldsymbol{S}_{n}$ can be computed from FFTs, where $\boldsymbol{O}_{m}$ and $\boldsymbol{S}_{n}$ are any observation and source vectors, respectively. For instance, the first term of equation (A15) is expanded as

$$
\begin{aligned}
& \left(f_{m} \rho_{m, x}^{p+}\right) G_{m, n}^{(1)}\left(\rho_{n, x}^{q+} f_{n}\right)+\left(f_{m} \rho_{m, y}^{p+}\right) G_{m, n}^{(1)}\left(\rho_{n, y}^{q+} f_{n}\right) \\
& +\left(f_{m} \rho_{m, z}^{p+}\right) G_{m, n}^{(1)}\left(\rho_{n, z}^{q+} f_{n}\right),
\end{aligned}
$$

where $f_{m, n}=1-w_{m, n}^{2} / 4$ and the subscripts $(x, y, z)$ stand for the components of the vector. The above equation is in a form such that the source point is on the right side of Green's function while the observation point is located on the left side of Green's function. In addition, three matrix-vector products are required, which are computed from FTTs. The same way is used for the last two terms of equation (A15), which requires 6 and 36 matrix-vector products, respectively, which are computed from FTTs. Thus, 45 matrix-vector products are necessary.

If the surface is not planar, then $R_{m, n}=$ $\sqrt{r_{m, n}^{2}+\left(z_{n}-z_{m}\right)^{2}}$, where $z(x, y)$ is the elevation of the surface. A Taylor series expansion over $z=z_{n}-z_{m}$ up to the fourth order leads to

$$
G_{m, n}^{(p)}=G_{m, n}^{(p),(0)}\left(1+z^{2} A_{m, n}^{(p),(1)}+z^{4} A_{m, n}^{(p),(2)}\right),
$$

where

$$
\left\{\begin{array}{l}
A_{m, n}^{(1),(1)}=-\frac{1+j u}{2 r_{m, n}^{2}} \\
A_{m, n}^{(2),(1)}=-\frac{2+j u}{2 r_{m, n}^{2}} \\
A_{m, n}^{(3),(1)}=-j \frac{u^{2}-5 j u-8}{2 r_{m, n}^{2}(u-2 j)}
\end{array}\right.
$$

$$
\left\{\begin{array}{l}
A_{m, n}^{(1),(2)}=\frac{3+3 j u-u^{2}}{8 r_{m, n}^{4}} \\
A_{m, n}^{(2),(2)}=\frac{8+5 j u-u^{2}}{8 r_{m, n}^{4}} \\
A_{m, n}^{(3),(2)}=\frac{9 j u^{2}+33 u-48 j-u^{3}}{8 r_{m, n}^{4}(u-2 j)}
\end{array}\right.
$$

and $G_{m, n}^{(p),(0)}=\left.G_{m, n}^{(p)}\right|_{z_{n}-z_{m}=0}=G_{m, n}^{(p)}\left(r_{m, n}\right), u=$ $k\left\|\boldsymbol{r}_{m, n}\right\|=k r_{m, n}\left(\boldsymbol{r}_{m, n}=\left(x_{n}-x_{m}\right) \hat{\boldsymbol{x}}+\left(y_{n}-\right.\right.$ $\left.\left.y_{m}\right) \hat{\boldsymbol{y}}\right)$. Since $\left\{A_{m, n}^{(p),(1)}, A_{m, n}^{(p),(2)}\right\}$ depends only on $r_{m, n}$, on a uniform grid, the matrices associated to the elements $\left\{G_{m, n}^{(p),(0)} A^{(p),(1)}, G_{m, n}^{(p),(0)} A^{(p),(2)}\right\}$ are also Toeplitz.

For the first order, since $R_{m, n}=r_{m, n}\left[1+z^{2} /\left(2 r_{m, n}^{2}\right)\right]$, the second order is neglected in comparison to the order zero if $k z^{2} /\left(2 r_{m, n}\right)<2 \pi / n_{0}$ (condition on the phase of $e^{-j k R_{m, n}}$ ) and if $z^{2} /\left(2 r_{m, n}^{2}\right)<1 / n_{0}$ (condition on the amplitude of $\left.1 / R_{m, n}\right)$, where $n_{0}>1$ is an integer ranging from 10 to 20 . Typically, $n_{0}=20$. This is consistent with equations (A20) and (A21). In other words, the strong interaction distance must satisfy

$$
r_{\text {Strong }}>\max \left(\frac{\max (\Delta z)^{2} n_{0}}{2 \lambda}, \max (\Delta z) \sqrt{\frac{n_{0}}{2}}\right),
$$

where $\Delta z=\max (z)-\min (z)$.

For a non-planar surface, the conditions $\boldsymbol{\delta}_{m, n}^{-}=-\boldsymbol{\delta}_{m, n}^{+}$and $\boldsymbol{\rho}_{m, n}^{p-, q-}=-\boldsymbol{\rho}_{m, n}^{p+, q+}$ are not satisfied. Then, the mean value is taken, that is $\boldsymbol{\delta}_{m, n}^{+} \rightarrow\left[\boldsymbol{\delta}_{m, n}^{+}+\left(-\boldsymbol{\delta}_{m, n}^{-}\right)\right] / 2$ and $\boldsymbol{\rho}_{m, n}^{p+q+} \rightarrow$ $\left[\boldsymbol{\rho}_{m, n}^{p+, q+}+\left(-\boldsymbol{\rho}_{m, n^{p-}}^{p-, q}\right)\right] / 2$.

\section{REFERENCES}

[1] S. Rao, D. Wilton, and A. Glisson, "Electromagnetic scattering by surfaces of arbitrary shape," IEEE Transactions on Antennas and Propagation, vol. 30, no. 409-18, 1982.

[2] W. C. Gibson, The Method of Moments in Electromagnetics, Chapman and Hall/CRC, Eds., London, 2008.

[3] A. Quarteroni, R. Sacco, and F. Sameri, Méthodes Numériques: Algortihmes, analyse et Applications. New-York: Springer-Verlag, 2007.

[4] L. Zhou, L. Tsang, V. Jandhyala, Q. Li, and C. H. Chan, "Emissivity simulations in passive microwave remote sensing with 3-D numerical solutions of Maxwell equations," IEEE Transactions on Geoscience and Remote Sensing, vol. 42, no. 8, pp. 1739-48, 2004. 
[5] S. W. Huang, G. H. Zhang, M. Y. Xia, and C. H. Chan, "Numerical analysis of scattering by dielectric random rough surfaces using modified SMCG scheme and curvilinear RWG basis functions," IEEE Transactions on Antennas and Propagation, vol. 57, no. 10, pp. 3392-3397, 2009.

[6] T.-H. Liao, L. Tsang, S. Huang, N. Niamsuwan, S. Jaruwatanadilok, S.-B. Kim, H. Ren, and K.-L. Chen, "Copolarized and cross-polarized backscattering from random rough soil surfaces from L-band to $\mathrm{Ku}$ band using numerical solutions of Maxwell's equations with near-field precondition," IEEE Transactions on Geoscience and Remote Sensing, vol. 54, no. 2, pp. 651-662, 2016.

[7] T. Qiao, L. Tsang, D. Vandemark, S. H. Yueh, T.-H. Liao, F. Nouguier, and B. Chapron, "Sea surface radar scattering at L-band based on numerical solution of Maxwell's equations in 3-D (NMM3D)," IEEE Transactions on Geoscience and Remote Sensing, vol. 56, no. 6, pp. 3137-47, 2018.

[8] E. Bleszynski, M. Bleszynski, and T. Jaroszewicz, "AIM: Adaptive integral method for solving large-scale electromagnetic scattering and radiation problems," Radio Science, vol. 31, no. 5, pp. 1225-1251, 1996.

[9] H. Bagci, A. E. Yilmaz, J.-M. Jin, and E. Michielssen, Modeling and Computations in Electromagnetics - Chapter 3: Time Domain Adaptive Integral Method for Surface Integral Equations, H. Ammari, Ed. Berlin: Springer, 2000

[10] W.-B. Ewe, L.-W. Li, and M.-S. Leong, "Solving mixed dielectric/conducting scattering problem using adaptive integral method," Progress In Electromagnetics Research, vol. 163, pp. 143-63, 2004.

[11] A. Colliander and P. Yla-Oijala, "Electromagnetic scattering from rough surface using single integral equation and adaptive integral method," IEEE Transactions on Antennas and Propagation, vol. 55, no. 12, pp. 3639-46, 2007.

[12] X. Wang, S.-X. Gong, J. Ling, and X.-M. Wang, "Interpolation scheme based on adaptive integral method for solving electrically large radiation problem by surface/surface configuration," Progress In Electromagnetics Research M, vol. 11, pp. 203-211, 2010

[13] X. Duan and M. Moghaddam, "3-D vector electromagnetic scattering from arbitrary random rough surfaces using Stabilized Extended Boundary Condition Method for remote sensing of soil moisture," IEEE Transactions on Geoscience and Remote Sensing, vol. 50, no. 1, pp. 87-103, 2012.

[14] R. Coifman, V. Rokhlin, and S. Wandzura, "The fast multipole method for the wave equation: a pedestrian prescription," IEEE Antennas and Propagation Magazine, vol. 35, no. 3, pp. 7-12, 1993.

[15] J. M. Song, C. C. Lu, and W. C. Chew, "Multilevel fast multipole algorithm for electromagnetic scattering by large complex objects," IEEE Transactions on Antennas and Propagation, vol. 45, no. 10, pp. 488 1493, 1997.

[16] D. Maystre, "Electromagnetic scattering from perfectly conducting rough surfaces in the resonance region," IEEE Transactions on Antennas and Propagation, vol. 31, no. 6, pp. 885-895, 1983.

[17] J. Hu and Z. Nie, "Improved electric field integral equation (IEFIE) for analysis of scattering from 3-D conducting structures," IEEE Transactions on Electromagnetic Compatibility, vol. 49, no. 3, pp. 644-48, 2007.

[18] C. C. Paige and M. A. Saunders, "An algorithm for sparse linear. equations and sparse least squares," ACM Transactions on Mathematical Software, vol. 8, no. 1, pp. 43-71, 1982.

[19] W. T. Sheng, Z. Y. Shu, K. Yang, and M. S. Tong, "Efficient evaluation of weakly singular integrals arising from electromagnetic surface integral equations," IEEE Transactions on Antennas and Propagation, vol. 61, no. 6, pp. 3377-3381, 2013.

[20] H. Braunisch, Y. Zhang, C. O. Ao, S.-E. Shih, Y. Eric Yang, K.-H. Ding, J. A. Kong, and L. Tsang, "Tapered wave with dominant polarization state for all angles of incidence," IEEE Transactions on Antennas and Propagation, vol. 48, no. 7, pp. 1086-1096, 2000.

[21] C. Bourlier, G. Kubické, and P. Pouliguen, "Accelerated computation of the physical optics approximation for near-field single- and doublebounces backscattering," IEEE Transactions on Antennas and Propagation, vol. 67, no. 12, pp. 7518-7527, 2019.

[22] C. Bourlier, "Acceleration of the computation of the method of moments EFIE impedance matrix from an updated fraunhofer criterion," IEEE Transactions on Antennas and Propagation, vol. Accepted for publication, Aug. 2020.

[23] M. Abramowitz and I. A. Stegun, Handbook of mathematical functions. New York: Dover publications, Inc., 1970. 